Mots. Les langages du politique

\title{
Agenda médiatique et élections présidentielles. L'à- propos des discours de campagne
}

Media agenda and presidential elections. Apropriateness of the campaign speeches

La agenda mediática y las elecciones presidenciales. El a propósito de los discursos de campaña

Julien Auboussier et Isabelle Huré

\section{OpenEdition}

Journals

Édition électronique

URL : https://journals.openedition.org/mots/22436

DOI : $10.4000 /$ mots.22436

ISSN : 1960-6001

Éditeur

ENS Éditions

\section{Édition imprimée}

Date de publication : 15 octobre 2016

Pagination : 45-52

ISBN : 978-2-84788-850-8

ISSN : 0243-6450

\section{Référence électronique}

Julien Auboussier et Isabelle Huré, « Agenda médiatique et élections présidentielles. L'à-propos des

discours de campagne », Mots. Les langages du politique [En ligne], 112 | 2016, mis en ligne le 15 octobre 2018, consulté le 22 avril 2022. URL : http://journals.openedition.org/mots/22436 ; DOI : https://doi.org/10.4000/mots.22436 


\section{Agenda médiatique et élections présidentielles. L’à-propos des discours de campagne}

Suffrage après suffrage, la question de l'influence des médias sur les comportements électoraux jouit d'une remarquable récurrence. Ainsi, la recherche a notamment interrogé la capacité des médias à décider l'ordre du jour des débats lors des campagnes électorales. En fixant l'agenda, c'est-à-dire en concentrant l'attention des citoyens sur un nombre limité de thématiques (ici considérées comme l'à propos des discours)'1, les médias pèseraient sur la façon dont les électeurs évaluent et comparent les candidats. C'est l'hypothèse portée, à partir des années soixante-dix aux États-Unis, par la théorie de l'agenda-setting: si les médias n'ont pas d'influence directe sur le choix du candidat par les électeurs, ils participeraient grandement à la focalisation de l'attention publique. Le développement des théories de l'agenda-setting aux ÉtatsUnis puis en Europe incite la recherche à prolonger le modèle initial par une meilleure prise en compte des interactions entre médias, personnel politique et opinion publique. C'est ce cheminement de la recherche, de la théorie initiale aux prolongements contemporains, que nous nous proposons ici de présenter.

\section{Les fondements de l'agenda-setting}

La théorie de l'agenda-setting est élaborée au début des années soixante-dix par Mc Combs et Shaw. Il s'agit alors d'interroger les conclusions de Lazarsfeld sur la campagne présidentielle de 1940 et le modèle du «two-step flow of communication » selon lequel les médias n'auraient que des effets faibles et indirects sur les choix de vote (Lazarsfeld et al., 1944).

L'étude fondatrice de Mc Combs et Shaw $(1972,1977)$ est menée à Chapel Hill en Caroline du Nord, lors de la campagne américaine de 1968. Les deux

1. Pour un approfondissement de la notion en analyse des textes et des discours : Ben Hamed, Mayaffre (2015).

Université de Franche-Comté, IUT Besançon-Vesoul, ELLIADD / DTEPS

auboussier_julien@hotmail.com

Université de Franche-Comté, UFR SLHS, ELLIADD / DTEPS

isabelle.hure@univ-fcomte.fr 
chercheurs partent du constat qu'en période électorale, les électeurs s’informent principalement par les médias. De là, ils font l'hypothèse d'une corrélation forte entre l'importance accordée à un sujet dans les discours médiatiques et la place qu'il occupe dans les préoccupations des électeurs. Pendant plusieurs semaines, ils comparent ainsi la façon dont les médias et les électeurs hiérarchisent les enjeux de la campagne en associant l'étude de l'agenda médiatique (via une analyse de contenu des discours à même de définir quantitativement les thématiques privilégiées) et des entretiens réguliers avec une centaine d'électeurs indécis.

Pour eux, le résultat est clair : l'intérêt des électeurs pour une thématique pendant la campagne dépend de la place que celle-ci occupe dans les médias à cette même période. Selon la fameuse formule, les médias ne nous diraient pas ce qu'il faut penser mais à quoi il faut penser ${ }^{2}$. En contexte électoral, les médias ne nous diraient donc pas pour qui voter mais sur quelles thématiques évaluer les candidats. En outre, en plus de participer à l'actualisation des préoccupations des électeurs au cours de la campagne, les médias influenceraient les candidats en les contraignant à se positionner sur les sujets prioritairement traités.

\section{Prolongements critiques}

L'étude fondatrice de Chapel Hill ouvre la voie à de nombreux travaux tant dans les pays anglo-américains (Weaver, 2007) qu'en France. Ceux-ci s'attachent notamment à dépasser les limites de ce modèle initial.

\section{Interroger les sources de l'agenda médiatique}

Certains travaux concernent l' «amont» de l'agenda médiatique. Ainsi, l'étude des pratiques des journalistes, et notamment celle de leurs sources (Schlesinger, 1992 ; Charon, 1995), contribue à renouveler les conceptions de l'agendasetting. En effet, " une des questions centrales auxquelles aboutit la recherche classique de l'agenda-setting est la suivante : qui détermine l'agenda des médias?» (Charon, 1995, p.74). Les discours des journalistes eux-mêmes ou ceux de leurs sources? Dans le cadre des campagnes électorales, la question ne cesse de gagner en acuité en raison de la professionnalisation de la communication politique dont l'objectif est, entre autres, de peser sur l'agenda médiatique et l'ordre du jour de la campagne.

La recherche attire aussi l'attention sur la concurrence qui règne entre les sources pour faire accéder telle ou telle thématique à l'agenda (Gerstlé, 1997,

2. "The press may not be successful much of the time in telling people what to think, but it is stunningly successful in telling its readers what to... think about » (Cohen, 1963, p.13; cité par Mc Combs, Shaw, 1972, p. 177). 
1999). L'accès aux médias exige en effet des ressources qui sont inégalement réparties entre les partis (partis majoritaires vs petits partis) ou entre acteurs sociaux (partis vs organisations de la société civile).

\section{Conjuguerl'agenda au pluriel}

D'autres travaux étoffent la théorie de l'agenda-setting en articulant l'agenda des médias, non plus seulement à celui des citoyens mais aussi à celui du personnel politique. Cette approche se développe sous l'impulsion de Bregman et Missika lors de leurs études des élections législatives de 1986 puis des élections présidentielles de 1988 (Bregman, Missika, 1986, 1988; Sawicki, 1991; Gerstlé, Duhamel, Davis, 1992).

Les résultats de l'enquête sur les élections de 1986 puis 1988 (Bregman, Missika, 1986, 1988) sont plus nuancés qu'à Chapel Hill. Il n'y a pas de fortes corrélations entre l'agenda médiatique et les résultats des sondages évaluant les priorités des électeurs. Ainsi, en 1986, alors que les médias nationaux se concentrent essentiellement sur le thème de la cohabitation, les citoyens s'intéressent prioritairement à l'emploi et à la crise économique. L'intérêt de l'étude est aussi sa prise en compte du travail journalistique. Ainsi, les chercheurs, en distinguant les "thèmes de préoccupations » des «thèmes de controverses » faisant l'objet d'oppositions entre les partis (ibid., p. 105), mettent en évidence l'appétence des médias pour la mise en scène des oppositions politiques au détriment du traitement en profondeur des thématiques. Les médias privilégient les sujets clivants afin de structurer et mettre en scène, souvent en la personnalisant, la compétition électorale.

D’un point de vue théorique, le médiacentrisme (Schlesinger, 1992) des premiers travaux est dépassé : «On se rend compte que l'étude des processus d'agenda repose sur le repérage des interactions réciproques qui régissent les relations entre trois types d'agenda : l'agenda des médias, l'agenda des citoyens et celui des acteurs politiques» (Bregman, 1989, p. 193). Les "effets d'agenda» (Bregman, Missika, 1988; Gerstlé, 2008) désignent dès lors la manière dont les agendas médiatique, politique et citoyen se construisent en interaction.

\section{Interroger les interactions entre les trois agendas}

Si la recherche insiste sur l'existence de plusieurs agendas, elle peine à évaluer leurs interactions au cours des campagnes électorales et leur hiérarchie. Un des trois agendas domine-t-il les autres? Les résultats sont souvent contradictoires et n'apportent pas de réponse définitive (Missika, 1989; Brandenburg, 2002 ; Veyrat-Masson, 2006; Vliegenthart, Walgrave, 2009).

Pour autant, l'idée selon laquelle les candidats sont soumis aux injonctions médiatiques en termes d'agenda est aujourd'hui contestée. Des études 
encouragent ainsi à penser les priorités des candidats en dehors du seul rapport entretenu à l'agenda des médias. Au-delà de l'anticipation des attentes de ces derniers, la logique et les enjeux liés au champ politique sont un paramètre essentiel de la sélection des controverses par les candidats (Sawicki, 1991; Gauthier, 1995 ; Crespin, 2006). C'est ainsi parl'analyse des dynamiques propres à l'espace politique que s'explique l'absence de la question de la protection sociale dans l'agenda des candidats lors de la campagne de 1988 (Sawicki, 1991). Plus récemment, Brouard, Grossman et Guinaudeau (2012) ont montré que l'existence des "petits partis», dont les programmes sont axés sur un thème précis comme l'écologie pour Les Verts, conduit le "grand parti» le plus proche idéologiquement - le PS - à s'approprier le thème. Par la suite, cette appropriation peut engendrer une assimilation du même thème parl'autre grand parti - l'UMP.

Ainsi, en interrogeant le poids du discours médiatique et en mettant au jour la diversité des agendas, les chercheurs ont tenté d'éloigner la théorie de l'agenda d'un fonctionnalisme réducteur pour adopter une orientation plus interactionniste à même de mieux rendre compte de la complexité de l'espace politique et journalistique. Il ne s'agit plus d'analyser le transfert de saillance des médias vers le public mais de penser la focalisation de l'attention publique à partir de l'interaction entre une diversité d'agendas.

\section{Prendre en compte le temps long du jeu politique}

D'autres études encore élargissent la durée des périodes d'observation en faisant l'hypothèse que la compréhension des processus par lesquels une thématique s'impose lors d'une campagne nécessite de dépasser le cadre de l'élection. Brugidou (1995), par exemple, montre qu'au moment des élections de 1988, l'Europe ne constitue qu' «un enjeu en voie de légitimation». Plus récemment, Mercier (2003) interroge l'idée partagée selon laquelle les médias auraient favorisé la présence de Jean-Marie Le Pen au second tour en 2002 par une médiatisation intensive de la thématique sécuritaire. Or, pour l'auteur, la présence de ce thème dans l'agenda politique et dans les médias précède largement l'élection. Il y voit essentiellement trois raisons : le vote en mai 2001 de la «loi sécurité» du gouvernement Jospin, le fait que le candidat Chirac se saisisse de la question dès juillet 2001 et en fasse un thème privilégié de campagne, et le poids de la sécurité dans les préoccupations des Français, révélé par divers sondages depuis le début des années quatre-vingt-dix. Autrement dit, si l'insécurité s'impose comme une thématique centrale dans la campagne de 2002, c'est le résultat, d'une part, de l'interaction entre les agendas de l'ensemble des politiques, de l'opinion publique et des médias et, d'autre part, d'une préoccupation latente qui dépasse le temps court de la campagne. L'analyste de discours ajoutera que le succès de la thématique tient aussi sans doute 
au pouvoir de la nomination : «insécurité » est une dénomination qui construit autant qu'elle décrit.

\section{Prendre en compte les discours et leurs dispositifs}

Les réserves quant à l'étude fondatrice de Mc Combs et Shaw ont enfin porté sur la pertinence des méthodes quantitatives (Bregman, 1989; Missika, 1989). La notion d'agenda n'épuise en effet pas les modalités d'influence du discours médiatique (Charon, 1995) et ne peut rendre compte de ce qui se joue dans le discours et par le discours. Qu'un sujet soit au cœur d'une campagne nous en dit peu sur la structuration du débat public. C'est à cette objection que répond le prolongement de la théorie de l'agenda par celle du cadrage développée aussi bien aux États-Unis qu'en Europe. Ainsi, après avoir relevé la saillance de la thématique du nucléaire, Brouard (et al., 2013) illustre la façon dont Nicolas Sarkozy, dans une stratégie de distinction vis-à-vis de ses concurrents, a privilégié un cadrage économique de cet enjeu. C'est aussi en termes d'agenda puis de cadrage que Belot (et al., 2013) analyse l'Europe comme thématique des discours des candidats lors de ces mêmes élections de 2012.

Au début des années quatre-vingt-dix, Mc Combs et Shaw (1993), tout comme un autre tenant de l'agenda-setting, David Weaver (1997), souscrivent à cette nouvelle orientation tout en insistant sur le fait que, pour eux, l'agendasetting reste le cadre général. Pourtant l'analyse des processus de cadrage semble dépasser l'analyse du seul transfert de focalisation et de l'ordre du jour pour ouvrir la voie à une analyse des dynamiques discursives qui alimentent les débats de campagne. Dès lors, la volonté des fondateurs de faire du cadrage le second niveau de l'agenda-setting ("second-level agenda-setting ») semble discutable. Ainsi, Emmanuel Marty (2015) propose une approche des discours de la presse régionale associant l'étude des cooccurrences lexicales à l'identification des cadres médiatiques privilégiés afin de mettre au jour les différents types de médiations journalistiques en période électorale.

C'est par ailleurs en étudiant les dispositifs d'énonciation et les formats médiatiques que des éléments essentiels dans la structuration des controverses ont été mis au jour. Par exemple, la durée de plus en plus réduite des extraits de discours des candidats diffusés aux informations télévisées ainsi que l'appétence des journalistes pour les «petites phrases» défavorisent la mise à l'agenda médiatique des questions complexes (Gerstlé, Piar, 2008) et, à l'inverse, favorisent un traitement des campagnes au prisme du conflit entre candidats (Gerstlé, Duhamel, Davis, 1992). 


\section{Les théories de l'agenda à l’ère numérique}

Aujourd'hui, les mutations de l'environnement médiatique et les évolutions concomitantes de la communication politique encouragent les chercheurs à réinterroger la pertinence de la notion d'agenda-setting. En 1993 déjà, Mc Combs et Shaw notaient: "Today two dozen newspaper racks are in front of the downtown post office, and an equal number of channels are available on cable television. The media system has fragmented ". Évidemment, l'avènement des blogs, des plateformes de partage et des réseaux sociaux a amplifié cette fragmentation de l'espace médiatique à laquelle doivent répondre les tenants des théories de l'agenda (Srömbäck, Kiousis, 2010). Ainsi, des études interrogent la façon dont Twitter (Vargo et al., 2013) ou Facebook (Freelon, 2013) ont participé à l'ordre du jour de la campagne américaine de 2012. Largement quantitatifs, ces travaux visent une meilleure compréhension à la fois de la nouvelle horizontalité de l'espace médiatique et de la remise en cause partielle de la hiérarchie traditionnelle des médias. Pour ce faire, certains adoptent, depuis peu, la notion d'agenda-melding (Shaw, Weaver, 2014), censée rendre compte des nouveaux comportements des citoyens face à l'information politique. La question devient alors : quelles sont les conséquences de la multiplication des sources d'information sur les effets d'agenda? Et notamment, dans quelle mesure l'information sur Internet pèse-t-elle sur l'agenda des médias traditionnels (Rebillard, 2012)?

Les méthodes d'objectivation thématique, notamment offertes par la lexicométrie, peuvent alimenter l'analyse sociologique de la configuration des agendas : toutes deux s'attachent en effet à l'identification de l'à propos des discours. Pour autant, leur inscription disciplinaire spécifique n'a pas encouragé leur convergence. Le prolongement de la notion d'agenda à celle de cadre a quelque peu changé la donne. Le discours politique n'est pas le lieu de la seule focalisation sur telle ou telle thématique, il est le lieu où se construisent des modes d'appréhension de la réalité sociale. Or, les cadres s'inscrivent dans la matérialité langagière et, en ce sens, peuvent être observés en discours. Ils sont constitués d'éléments (métaphores, schème argumentatif, routines dénominatives, formules, etc.) que le spécialiste des discours est à même d'identifier et d'analyser. Si agenda et cadre ne sont pas des concepts issus de l'analyse des discours politiques, force est de constater que les deux, surtout le second, peuvent être pensés depuis l'analyse de discours.

\section{Références}

BeLot Céline et al., 2013, "L'Europe comme enjeu clivant. Ses effets perturbateurs sur l'offre électorale et les orientations de vote lors de l'élection présidentielle de 2012 ", Revue française de science politique, vol. 63, nº, p. 1081-1112. 
Ben Hamed Mahé et MAYAffre Damon, 2015, « Les thèmes du discours. Du concept à la méthode ", Mots. Les langages du politique, $\mathrm{n}^{0} 108$, "Thèmes et thématiques dans le discours politique », p. 5-13.

Brandenburg Heinz, 2002, "Who follows whom? The impact of parties on media agenda formation in the 1997 British general elections campaign », Harvard Journal of Press and Politics, vol. 7, n03, p. 34-54.

BREgmAn Dorine, 1989, "La fonction d'agenda : une problématique en devenir », Hermès. Cognition, communication, politique, $\mathrm{n}^{\circ} 4$, p.191-202.

Bregman Dorine, MISSIKA Jean-Louis, 1986, «La campagne. La sélection des controverses politiques», Mars 1986, La drôle de défaire de la gauche, G. Grunberg, E. Dupoirier éd., Paris, PUF, p. 97-116.

- 1988, "Les priorités comparées des candidats et des médias », Le Monde. Dossiers et documents, mai 1988.

Brouard Sylvain, Grossman Emiliano, Guinaudeau Isabelle, 2012, "La compétition partisane française au prisme des priorités électorales. Compétition et appropriations thématiques », Revue française de science politique, vol. 62, nº 2, p. 255-276.

Brouard Sylvain, Gougou Florent, Guinaudeau Isabelle, Persico Simon, 2013, «Un effet de campagne. Le déclin de l'opposition des Français au nucléaire en 20112012 », Revue française de science politique, vol. 63, n 6, p. 1051-1079.

BRUgIdou Mathieu, 1995, L'élection présidentielle. Discours et enjeux politique, Paris, L'Harmattan.

Charron Jean, 1995, "Les médias et les sources. Les limites du modèle de l'agendasetting», Hermès, $\mathrm{n}^{\circ} 17-18, \mathrm{p} .73-92$.

CoвB Roger, Elder Charles, 1971, "The politics of agenda-building. An alternative perspective for modern democratic theory ", The Journal of Politics, vol. 33, n ${ }^{\circ} 4$, p. 892-915.

CRESPIN Renaud, 2006, «Drogues et sécurité routière. Changement politique ou nouvel usage des instruments? », Revue française de science politique, vol. 56, nº 5 , p. 813-836.

FreElon Deen, 2015, «Agenda-setting in the one-step flow. Evidence from Facebook in the 2012 election», 〈http://sites.bu.edu/cmcs/files/2015/03/Agenda-setting-inthe-one-step-flow.pdf $\rangle$ (consulté le 8 septembre 2015).

Gauthier Gilles, 1995, "L'analyse du contenu des débats politiques télévisés», Hermès, n' $17-18$, p. 355-370.

Gerstlé Jacques, 1997, «La persuasion de l’actualité télévisée», Politix, vol.10, n³, p. 81-96.

- 1999, "L'information et la sensibilité des électeurs à la conjoncture», Revue européenne des sciences sociales, nº114, p.139-157.

Gerstlé Jacques, Duhamel Olivier, Davis K. Dennis, 1992, «La couverture télévisée des campagnes présidentielles. L'élection de 1988 en France et aux États-Unis », Pouvoirs. Revue d'études constitutionnelles et politiques, nº 63, p. 53-69.

Gerstlé Jacques, PIAR Christophe, 2008, «Les campagnes de 2007 dans l'information télévisée. La structuration de l'agenda et ses conséquences", Le vote de rupture. Les élections présidentielles et législatives de 2007, P. Perrineau éd., Paris, Presses de Sciences Po, p. 21-50. 
Lazarsfeld Paul, Berelson Bernard R., Gaudet Hazel, 1944, The People’s Choice. How The Voter Makes up his Mind in a Presidential Campaign, New York, Columbia University Press.

MARTY Emmanuel, "Les élections municipales au miroir de la PQR. Des cadres médiatiques aux thématiques politiques», Mots. Les langages du politique, no ${ }^{108}$, «Thèmes et thématiques dans le discours politique », p. 39-54.

Mc Combs Maxwell E., SHAw Donald L., 1972, "The agenda-setting function of massmedia », Public Opinion Quarterly, vol. 36, n² 2, p. 176-187.

- 1993, "The evolution of agenda-setting research. 25 years in the marketplace of ideas ", Journal of Communication, vol. 43, $\mathrm{n}^{\circ} 2$, p. 58-67.

Mc Cомbs Maxwell E., SHAw Donald L. éd., 1977, The Emergence of American Political Issues. The Agenda-Setting Function of the Press, Eagan, West Publishing.

Mercier Arnaud, 2003, "Médias en campagne», Le vote de tous les refus, P. Perrineau éd., Paris, Presses de Sciences Po, p. 53-87.

MISSIKA Jean-Louis, 1989, "Les médias et la campagne présidentielle : autour de la notion de fonction d'agenda ", Études de communication. Langages, informations, médiations, nº10, p. 41-46.

SAWICKI Frédéric, 1991, "Les questions de la protection sociale dans la campagne présidentielle française de 1988 ", Revue française de science politique, vol.41, n² 2 , p. 171-196.

ShAw Donald J., WeAVER David, 2014, "Media agenda-setting and audience agendamelding ", Setting the agenda. The mass media and public opinion, M. Mc Combs éd., Cambridge, Polity Press, p. 145-150.

SCHLESINGER Philip, 1992, «Repenser la sociologie du journalisme. Les stratégies de la source d'information et les limites du média-centrisme », Réseaux. Communication, technologie, société, n5 51, p. 75-98.

StrömBÄCK Jesper, Kıousıs Spiro, 2010, «A new look at agenda-setting effects. Comparing the predictive power of overall political news consumption and specific news media consumption across different media channels and media types », Journal of Communication, vol.60, $\mathrm{n}^{\circ} 2$, p. 271-292.

RebilLARD Franck éd., 2012, «Internet et pluralisme de l'information», Réseaux. Communication, technologie, société, nº176, p. 9-25.

VARGo Chris, Guo Lei, Mc Combs Maxwell, SHAw Donald L., 2014, « Network issue agendas on Twitter during the 2012 US presidential election », Journal of Communication, vol.64, n² 2, p. 296-316.

VEYRAT-MASSON Isabelle, 2006, "Les recherches sur les campagnes électorales à la télévision », Le temps des médias. Revue d'histoire, nº 7, p. 289-301.

Vliegenthart Rens, Walgrave Stefaan, 2009, «Média et politique. Les conditions de l'effet des médias sur l'agenda parlementaire belge », Revue internationale de politique comparée, vol.16, n³, p. 423-440.

WeAVer David, 1997, «Framing should not supplant agenda-setting», Communication Theory \& Methodology (CT\&M) Concepts, vol. 27, nº 2, p. 3. 